\title{
Radiation in a Lossless Magneto-Ionic Medium at Frequencies High Relative to the Electron Gyro- frequency ${ }^{1}$
}

\author{
John W. Marini ${ }^{2}$
}

(Received March 27, 1963; revised June 13, 1963)

\begin{abstract}
Equations for the radiation field of an arbitrary distribution of source currents embedded in a lossless magneto-ionic medium and radiating at a frequency that is high relative to the electron gyrofrequency of the medium are derived using the $Q L$ approximation. The field is given directly in terms of the components of radiation vectors determined by an integration over the source currents. The application of the equations to arrays of similar sources is discussed.
\end{abstract}

\section{Introduction}

The subject of radiation from current sources in anisotropic media has received a great deal of attention in recent years. One reason for some of this activity is the current interest in space technology, plasma physics, and the problem of transmitting through the reentry plasma sheath [Bachynski, 1961].

Bunkin [1957] has published a formal solution for the radiation field of monochromatic current sources in an infinite homogeneous linear gyroelectric medium. In an earlier report Abraham [1953] attacked the problem of radiation from sources in a magneto-ionic medium by a somewhat different method. Since the publication of Bunkin's paper, a large number of papers related to this subject have appeared, including those of Chow [1962], Ford [1961], Kogelnik [1959], Kuehl [1962], and Shore and Meltz [1962].

The formal solution to the problem of electromagnetic radiation in gyroelectric media as given by Bunkin [1957] is incomplete in so far as it is necessary in order to obtain the field vectors, first to solve a transcendental equation [Bunkin, eq 3.13] and, second, to calculate the components of a matrix using this solution. While it does not appear to be feasible to obtain a general solution to the transcendental equation in question, solutions are possible in certain special cases. Bunkin, in the paper referenced, gave some results for the field of a dipole in two special directions, parallel to the static magnetic field of the medium and perpendicular to it. Kuehl [1962] has obtained solutions in several cases where special forms of the dielectric tensor are assumed. In one of these cases the limiting form at high frequency of the dielectric tensor of a lossless magneto-ionic medium (cold magnetoplasma) was used.

The subject of this paper is a case somewhat more general than the one for high frequency considered by Kuehl. While the same form of the dielectric tensor is assumed, and the relative gyrofrequency is likewise taken to be small, the relative plasma frequency is restricted only by the stipulation that the source frequency exceeds the plasma frequency. Also, terms of the first order in the relative gyrofrequency are retained in the expression obtained for the electromagnetic field. This expression is given in a form analogous to that commonly used [Schelkunoff, 1939] for the calculation of radiation from current sources in isotropic media. Both electric and magnetic type sources are considered, and the application of the expression to arrays of similar sources is discussed.

1 Work done in partial fulfillment of the requirements for the Ph. D. degree in Electrical Engineering at the University of Maryland. Dr. H. Reed acted as thesis advisor.

2 Present address: IIT Research Institute, Electromagnetic Compatability Analysis Center, USNMEL, Annapolis, Md. 


\section{Formal Āsymptotic Solution}

In this section the formal asymptotic solution for the field due to sources embedded in a lossless magneto-ionic medium is given.

\subsection{Maxwell's Equations}

In a magneto-ionic medium, Maxwell's equations for harmonic fields varying as exp $(j \omega t)$ are

$$
\begin{aligned}
& -j \omega \hat{\epsilon} E+\nabla \times H=J \\
& -\nabla \times E-j \omega \mu H=M .
\end{aligned}
$$

A fictitious magnetic current density $M$ is included [Schelkunoff, 1943] and rationalized mks units are used. $E, H$, and $J$ have their usual significance as the electric intensity, the magnetic intensity, and the electric current density respectively. The constants $\epsilon$ and $\mu$ are the electric permittivity of free space and the magnetic permittivity of free space respectively. The relative electric permittivity, $\hat{\epsilon}$, is a tensor. If Cartesian coordinates $x, y$, and $z$ are used with the $z$-axis directed along the static magnetic field in the medium, the tensor $\hat{\epsilon}$ takes the form [Suhl and Walker, 1954]:

$$
\hat{\boldsymbol{\epsilon}}=\left[\begin{array}{rrr}
a & -j b & 0 \\
j b & a & 0 \\
0 & 0 & c
\end{array}\right] .
$$

In the case under consideration, the quantities $a, b$, and $c$ are assumed to be given by the usual expressions in the magneto-ionic theory [Ratcliffe, 1959] with the collision frequency set equal to zero:

$$
\begin{aligned}
& a=1-X /\left(1-Y^{2}\right) \\
& b=X Y /\left(1-Y^{2}\right) \\
& c=1-X .
\end{aligned}
$$

where $X$ is the square of the relative electron plasma frequency, $X=\omega_{p}^{2} / \omega^{2}$, and where $Y$ is the relative gyrofrequency, $Y=\omega_{g} / \omega$.

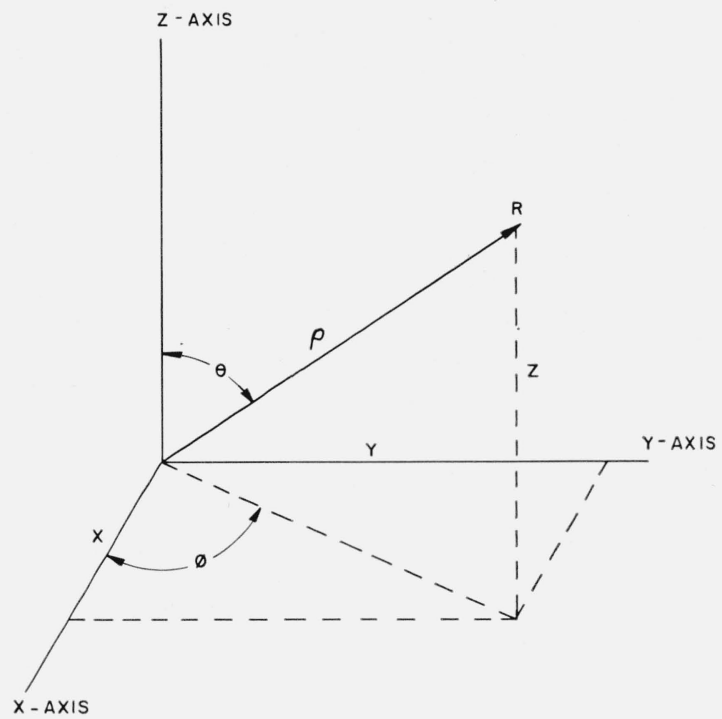

Figure 1. Components of radius vector $\mathrm{R}$.

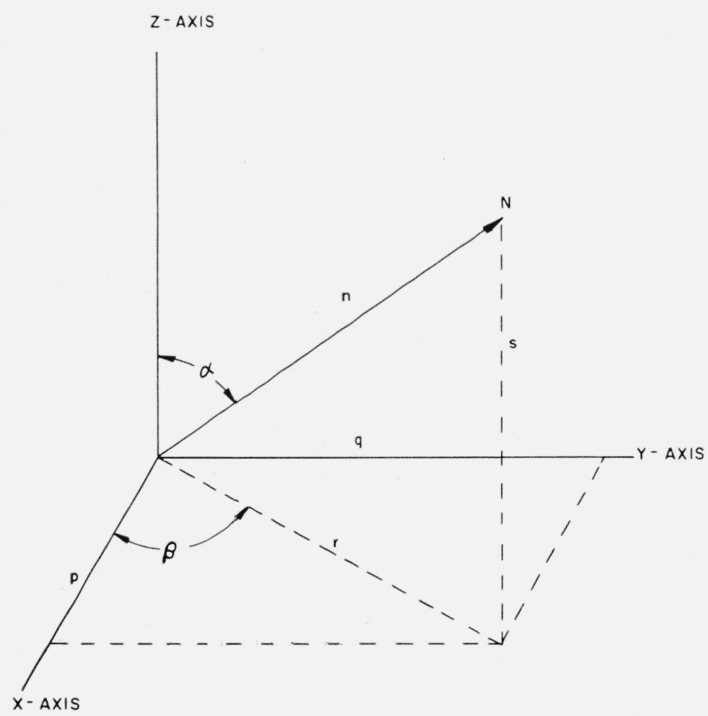

FIGURE 2. The components of the vector $\mathrm{N}$. 


\subsection{Method of Solution}

The components of $\hat{\epsilon}$ are independent of the coordinates in view of the assumed homogeneity of the medium, although they do depend on $\omega$. It is possible to obtain an asymptotic solution to (1) by using the Fourier transform pair

$$
\begin{aligned}
& \bar{f}(N)=\iint_{-\infty}^{+\infty} \int_{0} f(R) e^{j k N \cdot R} d x d y d z \\
& f(R)=\frac{k^{3}}{(2 \pi)^{3}} \iint_{-\infty}^{+\infty} \int_{\bar{f}(N) e^{-j k N \cdot R} d p d q d s}
\end{aligned}
$$

in which $R$ is the radius vector with Cartesian components $x, y$, and $z$ as shown in figure 1 , in which $N$ is a vector whose Cartesian components are the real parameters $p, q$, and $s$ as shown in figure 2 , and in which $N \cdot R$ is the scalar product of these two vectors. The transform pair (4) is valid for any positive real value of the constant $k$. Here $k$ is taken equal to $\omega \sqrt{\mu \epsilon}$.

The solution of (1) is accomplished by applying the transform (4a) to both sides of (1a) and of $(1 \mathrm{~b})^{3}$ to obtain a set of six algebraic equations in six unknowns

$$
\left[\begin{array}{rrrrrr}
a & -j b & 0 & 0 & -s & +q \\
j b & a & 0 & +s & 0 & -p \\
0 & 0 & c & -q & +p & 0 \\
0 & +s & -q & 1 & 0 & 0 \\
-s & 0 & +p & 0 & 1 & 0 \\
+q & -p & 0 & 0 & 0 & 1
\end{array}\right]\left[\begin{array}{c}
\bar{E}_{x} \\
\bar{E}_{y} \\
\bar{E}_{z} \\
\eta \bar{H}_{x} \\
\eta \bar{H}_{y} \\
\eta \bar{H}_{z}
\end{array}\right]=\left[\begin{array}{l}
-\bar{J}_{x} / j \omega \epsilon \\
-\bar{J}_{y} / j \omega \epsilon \\
-\bar{J}_{z} / j \omega \epsilon \\
-\bar{M}_{x} / j k \\
-\bar{M}_{y} / j k \\
-\bar{M}_{z} / j k
\end{array}\right]
$$

where $\eta=\sqrt{\mu / \epsilon}$. By using partitioned matrices, eq (5) can be abbreviated as

where $\hat{N}$ is defined as

$$
\left[\begin{array}{rr}
\hat{\epsilon} & \hat{N} \\
-\hat{N} & \hat{I}
\end{array}\right]\left[\begin{array}{c}
\bar{E} \\
\bar{\eta}
\end{array}\right]=\left[\begin{array}{l}
-\bar{J} / j \omega \epsilon \\
-\bar{M} / j k
\end{array}\right]
$$

and $\hat{I}$ is the identity matrix.

$$
\hat{N} \equiv\left[\begin{array}{rrr}
0 & -s & +q \\
+s & 0 & -p \\
-q & +p & 0
\end{array}\right]
$$

The next step in the solution is to premultiply (6) by the inverse of the dimensionless matrix

$$
\left[\begin{array}{rr}
\hat{\epsilon} & \hat{N} \\
-\hat{N} & \hat{I}
\end{array}\right]
$$

This inverse can be written as

$$
\left[\begin{array}{rr}
\hat{\epsilon} & \hat{N} \\
-\hat{N} & \hat{I}
\end{array}\right]^{-1}=\frac{1}{\Delta}\left[\begin{array}{ll}
\hat{A}(p, q, s) & \hat{B}(p, q, s) \\
\hat{C}(p, q, s) & \hat{D}(p, q, s)
\end{array}\right]
$$

\footnotetext{
${ }_{3}$ The usual procedure of first eliminating the vector $H$ from the pair of equations does not simplify the algebraic computations.
} 
where the matrix on the right-hand side of (9) is the adjoint of the matrix (8) and $\Delta$ is the determinant of the matrix (8). ${ }^{4}$ The form of $\hat{A}$ has been given elsewhere [Bunkin, 1957, eq 5.3; Kogelnik, 1959, eq 21]. $\hat{B}, \hat{C}$, and $\hat{D}$ can be obtained by matrix multiplication [Marini, 1963]: $\hat{B}=-\hat{A} \hat{N}, \hat{C}=+\hat{N} \hat{A}$, and $\hat{D}=\hat{N} \hat{B}+\Delta \hat{I}$.

The transform (4b) is then applied, giving formal integral expressions for $E$ and $H$. The latter integrals can be evaluated by the method of residues and the method of steepest descent to give the radiation field. The procedure for this evaluation has been discussed elsewhere [Bunkin, 1957; Lighthill, 1960; Chow, 1962; Shore and Meltz, 1962] and will not be repeated here. The final expressions for the radiation field are given in the next section.

\subsection{Formal Solution for the Radiation Field}

In Cartesian coordinates the complete formal solution for the radiation field is

$$
\begin{aligned}
& E(R)=\left[j \omega \mu \hat{A}_{0} V_{0}+j k \hat{B}_{0} W_{0}\right] g_{0} \frac{e^{-j k N_{0}(R) \cdot R}}{\rho}+\left[j \omega \mu \hat{A}_{e} V_{e}+j k \hat{B}_{e} W_{e}\right] g_{e} \frac{e^{-j k N_{e}(R) \cdot R}}{\rho} \\
& H(R)=\left[j k \hat{C}_{0} V_{0}+j \omega \epsilon \hat{D}_{0} W_{0}\right] g_{0} \frac{e^{-j k N_{0}(R) \cdot R}}{\rho}+\left[j k \hat{C}_{e} V_{e}+j \omega \epsilon \hat{D}_{e} W_{e}\right] g_{e} \frac{e^{-j k N_{e}(R) \cdot R}}{\rho}
\end{aligned}
$$

where

$g_{0, e}=\frac{ \pm \cos \theta}{4 \pi \cos \alpha_{0, e}} \mid \frac{n_{0, e} \sin \alpha_{0, e}}{\sin \theta \cos ^{2}\left(\alpha_{0, e}-\theta\right)\left[\left(n_{0, e}^{\prime \prime}-n_{0, e}\right) \cos \left(\alpha_{0, e}-\theta\right)-2 n_{0, e}^{\prime} \sin \left(\alpha_{0, e}-\theta\right)\right]}$

and where

$$
\left.\frac{1}{\left[(c-a)^{2} n_{0, e}^{4} \sin ^{4} \alpha_{0, e}-4 b^{2} c\left(n_{0, e}^{2} \sin ^{2} \alpha_{0, e}-c\right)\right]}\right|^{\frac{1}{2}}
$$

$$
\begin{aligned}
\hat{A}_{0, e} & =\hat{A}\left[p_{0, e}, q_{0, e}, s_{0, e}\right] \\
\hat{B}_{0, e} & =\hat{B}\left[p_{0, e}, q_{0, e}, s_{0, e}\right] \\
\hat{C}_{0, e} & =\hat{C}\left[p_{0, e}, q_{0, e}, s_{0, e}\right] \\
\hat{D}_{0, e} & =\hat{D}\left[p_{0, e}, q_{0, e}, s_{0, e}\right] .
\end{aligned}
$$

In eq (11) the lengths $n_{0}\left(\alpha_{0}\right)$ and $n_{e}\left(\alpha_{e}\right)$ of the vectors $N_{0}(R)$ and $N_{e}(R)$ are obtained from the formula for the index of refraction ${ }^{5}$

$$
n_{0, e}^{2}(\alpha)=\frac{2 a c+\left(a^{2}-b^{2}-a c\right) \sin ^{2} \alpha \pm \sqrt{\left(a^{2}-b^{2}-a c\right)^{2} \sin ^{4} \alpha+4 b^{2} c^{2} \cos ^{2} \alpha}}{2\left(a \sin ^{2} \alpha+c \cos ^{2} \alpha\right)}
$$

In eq (12) the Cartesian components $p_{0}, q_{0}, s_{0}$ and $p_{e}, q_{e}, s_{e}$ of the vectors $N_{0}(R)$ and $N_{e}(R)$ are obtained from their spherical components

$$
\begin{aligned}
& p_{0, e}=n_{0, e}\left(\alpha_{0, e}\right) \sin \alpha_{0, e} \cos \beta_{0, e} \\
& q_{0, e}=n_{0, e}\left(\alpha_{0, e}\right) \sin \alpha_{0, e} \sin \beta_{0, e} \\
& r_{0, e}=n_{0, e}\left(\alpha_{0, e}\right) \sin \alpha_{0, e} \\
& s_{0, e}=n_{0, e}\left(\alpha_{0, e}\right) \cos \alpha_{0, e} .
\end{aligned}
$$

${ }^{4} \Delta$ is also equal to the determinant of the matrix $\hat{\epsilon}+\hat{N}^{2}$ which premultiplies $\bar{E}$ when the usual procedure of first eliminating the vector $\bar{H}$ from eq (1) before applying the transform (4a) is followed.

${ }^{5}$ In eqs (11) and (13) the subscript 0 corresponds to the upper sign while the $e$ corresponds to the lower one. The abbreviations 0 and $e$ (for "ordinary" and "extraordinary") can be used here since the only case under consideration is the one where the quantities $a, b$, and $c$ are given by eq (3) and a frequency higher than the plasma frequency is used [Ratcliffe, 1959, sec. 7.3]. 
$V_{0, e}$ and $W_{0, e}$ are radiation vectors

$$
\begin{aligned}
V_{0, e} & =\iint_{-\infty}^{+\infty} \int_{0}^{j k N_{0, e}(R) \cdot R^{\prime}} J\left(R^{\prime}\right) d x^{\prime} d y^{\prime} d z^{\prime} \\
W_{0, e} & =\iint_{-\infty}^{+\infty} \int_{-} e^{j k N_{0, e}(R) \cdot R^{\prime}} M\left(R^{\prime}\right) d x^{\prime} d y^{\prime} d z^{\prime}
\end{aligned}
$$

calculated by a direct integration over the current sources.

The solution given in eqs (10-16) above depends entirely on the values assigned to the azimuthal angles $\beta_{0}$ and $\beta_{e}$, and to the polar angles $\alpha_{0}$ and $\alpha_{e}$. These are determined from the saddle point evaluation, which gives

and

$$
\beta_{0, e}=\varphi
$$

$$
n^{\prime}\left(\alpha_{0, e}\right) / n\left(\alpha_{0, e}\right)=\tan \left(\alpha_{0, e}-\theta\right) \text {. }
$$

Equation (18) is the transcendental equation mentioned in the introduction upon which the entire solution depends. Kuehl [1962, eq (13)] has given explicitly the form taken by this equation when $n_{0, e}(\alpha)$ given in (13) is substituted into it. If this form is specialized to the case of a lossless magneto-ionic medium by using the values given in eq (3) for the quantities ${ }^{6}$ $a, b$, and $c$, the eq (18) takes the form ${ }^{7}$

$$
\tan \left(\alpha_{0, e}-\theta\right)=\frac{\mp X Y^{2} \sin \alpha_{0, e} \cos \alpha_{0, e}}{2 m^{2} v\left(\alpha_{0, e}\right)\left[1-Y^{2} \sin ^{2} \alpha_{0, e} / 2 m^{2} \pm v\left(\alpha_{0, e}\right)\right]} .
$$

In (19) above the abbreviations

$$
\begin{gathered}
m^{2}=1-X \\
v(\alpha)=\sqrt{Y^{4} \sin ^{4} \alpha / 4 m^{4}+Y^{2} \cos ^{2} \alpha}
\end{gathered}
$$

have been used. The quantity $m$ may be interpreted as the length of the vectors $N_{0, e}$ if the static magnetic field in the magneto-ionic medium is set equal to zero.

If the values given in eq (3) are used in (13), then the Appleton-Hartree formula for the index of refraction in a lossless magneto-ionic medium results:

$$
n_{0, e}^{2}(\alpha)=1-X /\left[1-Y^{2} \sin ^{2} \alpha / 2 m^{2} \pm v(\alpha)\right] .
$$

\section{Approximate Solution}

In this section the method of approximation used to obtain a solution to eq (19) will be indicated, and the resulting equations for the electromagnetic field will be given.

\subsection{Method of Approximation}

As mentioned previously, only the case where the wave frequency is high relative to the gyromagnetic frequency of the medium will be treated. Specifically it will be assumed that $Y$ is small enough to permit the simplification of (19) to be carried out by expanding all quantities in powers of $Y$ and dropping high order terms.

In the case of an isotropic medium with $Y$ equal to zero, the right-hand side of (19) vanishes and $\alpha_{0, e}$ can assume only the single value $\theta$, which is the correct result. In the case under

\footnotetext{
${ }_{6}$ The algebra is simplified if use is first made of the relation $a^{2}-b^{2}-a c=a-c$ that exists between the quantities $a, b$, and $c$ defined in (3).

7 A factor $\omega_{g} / \omega$ appears to be missing from the corresponding equation given by Kuehl [1962, eq (46)].
} 
consideration, consequently, it is assumed that the angles $\alpha_{0, e}$ deviate only slightly from the limiting value $\theta$ and that the deviation $\alpha_{0, e}-\theta$, has an order of magnitude no larger than that of $Y$. In view of the expansion

$$
\tan \left(\alpha_{0, e}-\theta\right)=\alpha_{0, e}-\theta+\left(\alpha_{0, e}-\theta\right)^{3} / 3+\ldots
$$

eq (19) can be approximated by

$$
\alpha_{0, e}=\theta \mp \frac{X Y^{2} \sin \alpha_{0, e} \cos \alpha_{0, e}}{2 m^{2} v\left(\alpha_{0, e}\right)\left[1-Y^{2} \sin ^{2} \alpha_{0, e} / 2 m^{2} \pm v\left(\alpha_{0, e}\right)\right]} .
$$

It remains to approximate $\sin \alpha_{0, e}, \cos \alpha_{0, e}$, and $v\left(\alpha_{0, e}\right)$. The first two are approximated by writing $\alpha_{0, e}$ as $\alpha_{0, e}=\theta+\left(\alpha_{0, e}-\theta\right)$ and using appropriate trigonometric expansions.

Some care must be exercised, however, when the quantity $v(\alpha)$ defined in (21) is approximated. For most values of $\alpha$, the approximation

$$
v(\alpha) \cong Y|\cos \alpha|
$$

can be made, since the first term under the radical contains $Y$ to the fourth power. If $\alpha$ is very close to $\pi / 2$, nevertheless, then the approximate value of (21) becomes

$$
v(\alpha) \cong Y \sin ^{2} \alpha / 2 m^{2}+m^{2} \cot ^{2} \alpha .
$$

Equations (25) and (26) correspond to the $Q L$ and $Q T$ approximations respectively of the magneto-ionic theory [Ratcliffe, 1959, ch. 8]. In view of the assumption that $Y$ is small, the $Q L$ approximation will hold over most of the region surrounding the sources. The $Q T$ approximation is valid only over a small angular region in which rays from the sources are approximately perpendicular to the static magnetic field. For this reason only the treatment of the QL case is included in this paper, although the $Q T$ case also can be handled in the same way [Marini, 1963].

\subsection{Components of $N_{0, e}(R)$}

The resulting approximate solution to (19) for the polar angle of $N_{0, e}(R)$ in terms of the polar angle of $R$ is

$$
\alpha_{0, e}=\theta \mp X Y \sin \theta \cos \theta / 2 m^{2}|\cos \theta| .
$$

An additional term can easily be obtained in the expression (27) for $\alpha_{0, e}$ but the accuracy given is sufficient for present purposes.

Using (27) above in (22), the length of $N_{0, e}(R)$ is found to be given by

$$
n_{0, e}^{2}=m^{2}\left[1 \pm \frac{X Y}{m^{2}}|\cos \theta|-\frac{X Y^{2}}{2 m^{2}}\left(1+\cos ^{2} \theta\right)\right]
$$

from which

$$
n_{0, e}=m\left\{1 \pm \frac{X Y}{2 m^{2}}|\cos \theta|-\frac{X Y^{2}}{4 m^{4}}\left[m^{2}+\left(1-\frac{X}{2}\right) \cos ^{2} \theta\right]\right\}
$$

The azimuthal angle of $N_{0, e}(R)$ is, as previously mentioned,

$$
\beta_{0, e}=\varphi \text {. }
$$

Equations (27), (29), and (30) above give approximate values for the spherical polar components of $N_{0}(R)$ and $N_{e}(R)$ in terms of the spherical polar components of $R$. The Cartesian components are found from (14). 


\subsection{The Radiation Field}

The approximate equations for the radiation field can now be obtained by substituting the approximate values of the components of $N_{0, e}(R)$ found in the preceding section into eqs (10), (11), (12), (15), and (16). The components of the field vectors will be in Cartesian coordinates.

It is evident that the matrices $\hat{A}_{0, e}, \hat{B}_{0, e}, \hat{C}_{0, e}$, and $\hat{D}_{0, e}$ are the Cartesian components of the tensors that relate the components of the radiation vectors to those of the field vectors. The use of Cartesian coordinates was dictated principally by the fact that the components of $\hat{\epsilon}$ can be made to assume the simple form (2) in that system of coordinates. Kuehl [1961] has shown, however, that the equations for the electromagnetic field are more compact if coordinate systems based on the planes of constant phase of the radiated waves are used. In the case under consideration, with $\alpha_{0, e}-\theta$ small, almost equal simplification is obtained by using spherical polar coordinates, and this will be done in what follows.

One more simplification can be made. The absolute value sign that appears in (27) and (28) can be removed. This necessitates a change in notation, however, since the identity of the ordinary and extraordinary wave is switched in going from the upper half to the lower balf space.

With these changes, with the vector electric intensity written in spherical polar coordinates as

$$
\mathbf{E}(R)=\left[\begin{array}{l}
E_{\rho} \\
E_{\theta} \\
E_{\phi}
\end{array}\right]
$$

and with a like notation used for the magnetic intensity, the radiation vectors, and the current densities, the equations for the field vectors are

$$
\begin{aligned}
\mathbf{E}(R) & =(j / 8 \pi \rho)\left[\omega \mu \hat{\mathbf{A}}_{1} \mathbf{V}_{1}+k \hat{\mathbf{B}}_{1} \mathbf{W}_{1}\right] e^{-j k N_{1}(R) \cdot R}+(j / 8 \pi \rho)\left[\omega \mu \hat{\mathbf{A}}_{2} \mathbf{V}_{2}+k \hat{\mathbf{B}}_{2} \mathbf{W}_{2}\right] e^{-j k N_{2}(R) \cdot R} \\
\mathbf{H}(R) & =(j / 8 \pi \rho)\left[k \hat{\mathbf{C}}_{1} \mathbf{V}_{1}+\omega \epsilon \hat{\mathbf{D}}_{1} \mathbf{W}_{1}\right] e^{-j k N_{1}(R) \cdot R}+(j / 8 \pi \rho)\left[k \hat{\mathbf{C}}_{2} \mathbf{V}_{2}+\omega \epsilon \hat{\mathbf{D}}_{2} \mathbf{W}_{2}\right] e^{-j k N_{2}(R) \cdot R}
\end{aligned}
$$

where $\hat{\mathbf{A}}_{1,2}, \hat{\mathbf{B}}_{1,2}, \hat{\mathbf{C}}_{1,2}$, and $\hat{\mathbf{D}}_{1,2}$ are given by

$$
\begin{aligned}
& \hat{\mathbf{A}}_{1,2}=\left[\begin{array}{ccc}
0 ; & \pm h \sin \theta ; & -j h \sin \theta \\
\pm h \sin \theta ; & -1 \pm h\left(\cos \theta-\sin ^{2} \theta / X \cos \theta\right) ; & \pm j-j h \cos \theta \\
j h \sin \theta ; & \mp j+j h \cos \theta ; & -1 \pm h\left(\cos \theta+\sin ^{2} \theta / X \cos \theta\right)
\end{array}\right] \\
& \hat{\mathbf{B}}_{1,2}=m\left[\begin{array}{ccc}
0 ; & j h \sin \theta ; & \pm h \sin \theta \\
-j h \sin \theta ; & \mp j ; & -1 \mp h \sin ^{2} \theta / X \cos \theta \\
\pm h \sin \theta ; & 1 \mp h \sin ^{2} \theta / X \cos \theta ; & \mp j
\end{array}\right] \\
& \hat{\mathbf{C}}_{1,2}=m\left[\begin{array}{ccc}
0 ; & j h \sin \theta ; & \pm h \sin \theta \\
-j h \sin \theta ; & \pm j ; & 1 \mp h \sin ^{2} \theta / X \cos \theta \\
\pm h \sin \theta ; & -1 \mp h \sin ^{2} \theta / X \cos \theta ; & \pm j
\end{array}\right]
\end{aligned}
$$

and

$$
\hat{\mathbf{D}}_{1,2}=m^{2}\left[\begin{array}{ccc}
0 ; & \mp h \sin \theta ; & j h \sin \theta \\
\mp h \sin \theta ; & -1 \mp h\left(\cos \theta-\sin ^{2} \theta / X \cos \theta\right) ; & \pm j+j h \cos \theta \\
-j h \sin \theta ; & \mp j-j h \cos \theta ; & -1 \mp h\left(\cos \theta+\sin ^{2} \theta / X \cos \theta\right)
\end{array}\right]
$$


with $h$ defined as

$$
h=X Y / 2 m^{2} .
$$

In the equations above, the upper sign is always associated with index 1 and the lower sign with the index 2. Here, however, the terms associated with the former index represent the ordinary wave when $\theta<90^{\circ}$, but represent the extraordinary wave when $\theta>90^{\circ}$.

The exponents in (32) above are

$$
-j k N_{1,2}(R) \cdot R=-j k \rho m(1 \pm h \cos \theta)
$$

and the spherical polar components of the radiation vectors are calculated from those of the source currents through

and

$$
\mathbf{V}_{1,2}(R)=\iiint \mathbf{J}\left(R^{\prime}\right) e^{j k N_{1,2}(R) \cdot R^{\prime}} \rho^{\prime 2} \sin \theta^{\prime} d \rho^{\prime} d \theta^{\prime} d \varphi^{\prime}
$$

$$
\mathbf{W}_{1,2}(R)=\iiint \mathbf{M}\left(R^{\prime}\right) e^{j k N_{1,2}(R) \cdot R^{\prime}} \rho^{\prime 2} \sin \theta^{\prime} d \rho^{\prime} d \theta^{\prime} d \varphi^{\prime}
$$

with the exponent given by

$$
\begin{aligned}
j k N_{1,2}(R) \cdot R^{\prime} & =j k \rho^{\prime} m\left[\sin \theta^{\prime} \sin \theta \cos \left(\varphi^{\prime}-\varphi\right)+\cos \theta^{\prime} \cos \theta \pm h \cos \theta^{\prime}\right] \\
& =j k \rho^{\prime} m \cos \psi \pm j k m h z^{\prime},
\end{aligned}
$$

where $\psi$ is the angle between $R$ and $R^{\prime}$.

Equations (32) through (40) constitute the desired approximate solution to the problem of calculating the radiation field of given source currents fixed in a lossless magneto-ionic medium and radiating at a frequency above the plasma frequency and well above the gyrofrequency.

\section{Application of the Solution to Radiation From Arrays}

The first order $Q L$ solution (32) holds over most of the region surrounding the source currents when the assumed conditions are met. It is interesting to consider the significance of this solution as it applies to the radiation from an array of similar sources. For brevity, only electric type sources are discussed.

The current density in an array of $n$ sources located at $R_{1}, R_{2}, \ldots R_{n}$ can be written as

$$
J(R)=\sum_{i=1}^{n} f_{i} J_{s}\left(R-R_{i}\right)
$$

where $J_{s}(R)$ represents the current density of one of the sources, and the complex constants $f_{i}$ are feeding coefficients that describe the relative amplitudes and phases between the sources. Substituting into (39), the radiation vectors for such an array are

$$
\mathbf{V}_{1,2}(R)=\sum f_{i} e^{j k \rho_{i} m \cos \psi_{i} \pm j k m h z_{i}} \cdot \iiint J_{s}\left(R^{\prime}\right) e^{j \kappa_{\rho^{\prime}} m \cos \psi \pm j k m h z^{\prime}} \rho^{\prime 2} \sin \theta^{\prime} d \rho^{\prime} d \theta^{\prime} d \phi^{\prime} .
$$

The existence of the two radiation vectors $V_{1}$ and $V_{2}$ above indicates that the patterns of the two characteristic waves emitted by the array might, in general, just as well be treated separately. However, when the array is so limited in size or is so oriented that the term $(k m h z)$ produces negligible change in phase over the array and its sources, then the radiation vectors $V_{1}$ and $V_{2}$ are equal, and the electric intensity (32a) can be written as

with

$$
\mathbf{E}(R)=\frac{j \omega \mu}{4 \pi \rho} e^{-j k \rho m}\left[\cos (k \rho m h \cos \theta)\left(\hat{A_{1}}+\hat{A_{2}}\right) / 2-j \sin (k \rho m h \cos \theta)\left(\hat{A_{1}}-\hat{A_{2}}\right) / 2\right] \mathbf{V}
$$

$$
\mathbf{V}=\sum f_{i} e^{j k_{\rho_{i}} m \cos \psi_{i}} \cdot \iiint J_{s}\left(R^{\prime}\right) e^{j k_{\rho^{\prime}} m \cos \psi} \rho^{\prime 2} \sin \theta^{\prime} d \rho^{\prime} d \theta^{\prime} d \phi^{\prime} .
$$


The eq (44) for the radiation vector $\mathbf{V}$ above is identical with the formula that would give the radiation vector of the array it it were immersed in an isotropic medium having an index of refraction equal to $m$. It is equal to the product of the complex space factor of the array [Schelkunoff, 1943, sec. 9.3] times the radiation vector of the source. From this it follows that a principle of pattern multiplication [Kraus, 1950] can be stated for the array considered above as follows:

In a lossless magneto-ionic medium at frequencies above the plasma frequency and well above the gyrofrequency, the total field pattern of an array composed of similar sources and over which a phase change of magnitude $(\mathrm{kmh})$ radians per unit length in the $z$ direction can be neglected is equal to the product of the pattern of an array of point sources calculated by neglecting the effect of the static magnetic field and the pattern of an individual source in the anisotropic medium. The electric intensity from which the latter pattern is calculated is given by (43) using the radiation vector of an individual source in place of $\mathbf{V}$. This electric intensity differs from that existing in an isotropic medium both because of the factors $\cos (k \rho m h \cos \theta)$ and $\sin (k \rho m h \cos \theta)$ appearing in (43) and because of the presence of terms containing the factor $h$ in the matrices $\hat{A}_{1}$ and $\hat{A}_{2}$ in (43). If the latter terms are small enough to be neglected, then the only effect of the anisotropy of the medium on the radiation field of the source and consequently of the entire array is the Faraday rotation due to the cosine and sine factors.

In dealing with arrays in which the phase shift due to the terms $\left(k m h z^{\prime}\right)$ in $(40)$ is not negligible, the two radiation vectors $V_{1}$ and $V_{2}$ are distinct. Consequently, the radiation patterns of the associated characteristic waves cannot be combined as was done above. However, a principle of pattern multiplication can still be stated for each characteristic wave. Since the term $\left(k m h z^{\prime}\right)$ is independent of the direction of $R$, the phase shift due to this term can be associated with that of the feeding coefficients of the array making the following statement possible:

Each characteristic wave emitted by an array of sources immersed in a lossless magnetoionic medium and radiating at a frequency above the plasma frequency and well above the gyrofrequency has a pattern equal to the product of the pattern of the characteristic wave emitted by an individual source times a space factor of the array. The latter can be calculated by assuming the array to be immersed in the plasma with no static magnetic field present, provided that the feeding coefficients of the array are changed by a compensating phase shift of $\pm \mathrm{kmh}$ radians per unit length in the $z$ direction.

\section{Conclusions}

The electromagnetic field radiated by either an electric or a magnetic type source embedded in a lossless magneto-ionic medium has been given directly in terms of the components of two radiation vectors. One of the vectors gives the field due to the ordinary wave while the other gives the field due to the extraordinary wave. The components of the radiation vectors are obtained by an integration over the source.

The approximations used to derive the solution restrict the application of the latter to cases where the source frequency is above the plasma frequency and is high compared to the gyromagnetic frequency of the medium. Also the relative geometrical placement between the source and the field point must be such that the $Q L$ approximation is valid. The AppletonHartree equations for a lossless magneto-ionic medium are used.

An examination of the solution shows that the principal effect of the anisotropy of the medium on the radiation field of a source of limited extent is a Faraday rotation of the field that would exist if the medium were made isotropic by setting its static magnetic field equal to zero. There is in addition, however, some first order distortion of the pattern of the source proportional to the strength of the static magnetic field. The space factor of the pattern of an array of similar sources is affected only by the incremental Faraday rotation between the elements of the array. When the extent of the array is such that this incremental rotation is negligible, the space factor of the array is identical with that obtained by treating the medium as isotropic. When the rotation is not negligible a distinct array space factor is obtained for 
each of the characteristic waves. These space factors can also be calculated, however, from the formulas for the pattern of the array in an isotropic medium by making compensating changes in the phases of the feeding coefficients of the array elements.

\section{References}

L. G. Abraham, Jr. (Aug. 1, 1953), Extensions of the magneto-ionic theory for radio-wave propagation in the ionosphere including antenna radiation and plane wave scattering, Cornell Univ. School of E. E. Tech. Rept. No. 13 on studies on propagation in the ionosphere.

M. P. Bachynski (Dec. 1961), Plasma physics — an elementary review, Proc. IRE 4, 1751-1766.

F. V. Bunkin (Feb. 1957), On radiation in anisotropic media, Soviet Phys.-JETP 32, 338-346.

Y. Chow (July 1962), A note on radiation in a gyro-electric-magnetic medium - an extension of Bunkin's calculation, IRE Trans. Ant. Prop. AP-10, 464-469.

G. W. Ford (1961), Electromagnetic radiation from a source in a plasma, Ann. Phys. 16, 185-200.

H. Kogelnik (Sept.-Oct. 1959), On electromagnetic radiation in magneto-ionic media, J. Res. NBS 64D (Radio Prop.), 515-522.

J. D. Kraus (1950), Antennas, sec. 4-3 (McGraw-Hill Book Co., Inc., New York, N.Y.).

H. H. Kuehl (Sept. 1962), Electromagnetic radiation from an electric dipole in a cold anisotropic plasma, Phys. Fluids 5, 1095-1103.

M. J. Lighthill (1960), Studies on magneto-hydrodynamic waves and other anisotropic wave motions, Phil. Trans. Roy. Soc. A252, 397-430.

J. W. Marini (1963), Radiation from sources in magneto-ionic media, Doctoral Thesis, University of Maryland. Obtainable from University Microfilms, Inc., 313 N. First St., Ann Arbor, Mich.

J. A. Ratcliffe (1959), The magneto-ionic theory and its applications to the ionosphere (Cambridge Univ. Press, New York, N.Y., and London, England).

S. A. Schelkunoff (Oct. 1939), A general radiation formula, Proc. IRE 27, 660-666.

S. A. Schelkunoff (1943), Electromagnetic waves, sections 4.1 and 4.4 (D. Van Nostrand Co., New York, N.Y.).

R. Shore and G. Meltz (Jan. 1962), Anisotropic plasma-covered magnetic line source, IRE Trans.-Ant. Prop. AP10, 78-82.

H. Suhl and L. R. Walker (May 1954), Topics in guided wave propagation through gyromagnetic media, Bell Sys. Tech. J. 33, 579-659.

\section{Additional Reference}

S. Ramo and J. R. Whinnery (1959), Fields and Waves in Modern Radio, Ch. 12 (John Wiley \& Sons, Inc., New York, N.Y.).

(Paper 67D6-300) 